\title{
Descrição do pupário de Sargus thoracicus Macquart (Diptera, Stratiomyidae, Sarginae)
}

\author{
Gustavo Glória Viana ${ }^{1,2}$ \\ Roberto de Xerez ${ }^{1,3}$
}

\begin{abstract}
Description of the puparium of Sargus thoracicus Macquart (Diptera, Sarginae, Stratiomyidae). Herein, the puparium of Sargus thoracicus Macquart, 1834 collected in decaying organic material, on the Campus of Universidade Federal Rural do Rio de Janeiro, Seropédica, Rio de Janeiro State is described. The description is based on a single larva collected and reared in laboratory till the emergence of the adult. Some biological notes on the larva are also presented.

KEY WORDS. decomposition, puparium, Sarginae, Sargus thoracicus
\end{abstract}

Os Stratiomyidae formam um grupo de moscas cosmopolitas com grande diversidade de formas, algumas espécies apresentando formas miméticas de vespas (KOVAC \& ROZKOŠNÝ 1997). As larvas podem ser aquáticas, semi-aquáticas ou terrestres, estando associadas a restos de frutos, verduras, troncos de mamoeiro e banana em decomposição, a raízes e folhas de plantas aquáticas ou ainda a troncos de árvores em decomposição (MCFADDEN 1967).

Larvas de Stratiomyidae podem ser reconhecidas pelas seguintes combinações de caracteres: 1) corpo dividido em 11 segmentos, exceto em Chiromyza vittata Widemann, 1830 onde são observados 12 segmentos em vista lateral e ventral (PUJOL-LUZ \& VIEIRA 2000); 2) cutícula com usual forma de mosaico, impregnada por carbonato de cálcio; 3) ornamentações cuticulares entre os segmentos e nas margens laterais do corpo; 4) espiráculos laterais no primeiro segmento torácico; em larvas aquáticas; 5 ) presença de um ou dois ganchos na região ventral do sexto e do sétimo segmento abdominal ou 1 par de ganchos no sétimo segmento abdominal e; 6) espiráculo posterior abrindo-se por um tufo de cerdas no oitavo segmento abdominal (ROZKOŠNÝ 1982).

Larvas de Sarginae são pouco conhecidas na região Neotropical devido a falta de conhecimento dos habitats e a dificuldade de se criar em laboratório larvas deste grupo de moscas. De acordo com MCFADDEN (1967), as larvas de Sarginae são coprófogas ou sapronecrofitófogas, participando do processo de reciclagem de material orgânico em decomposição. Larvas de Sarginae caracterizam-se por serem delgadas, de comprimento entre 5-15 mm, presença de cerdas bem desenvolvidas no complexo mandíbulo-maxilar e geralmente com um padrão de listas longitudinais (JAMES 1981).

1) Departamento de Biologia Animal, Instituto de Biologia, Universidade Federal Rural do Rio de Janeiro. Rodovia BR 465, Km 07, 23890-000 Seropédica, Rio de Janeiro, Brasil.

2) Bolsista PIBIC/CNPq. E-mail: ggviana@ufrrj.br

3) E-mail: rdexerez@ufrrj.br 
Os Sarginae possuem 23 gêneros distribuídos por todas as regiões zoogeográficas. O gênero Sargus Fabricius, 1798 possui 46 espécies distribuídas pela região Neotropical, sendo que 16 espécies foram citadas como ocorrentes no Brasil (WOODLEY 2001). Segundo J.R. Pujol Luz (comunicação pessoal) três ocorrem no Rio de Janeiro (Sargus brasiliensis Wiedemann, 1830, Sargus circuncinctus (James, 1941) e Sargus thoracicus). MCFADDEN (1967) descreveu larvas e pupários das seguintes espécies ocorrentes na região Neártica: Sargus elegans Loew, 1866, Sargus decorus Say, 1824, Sargus viridis Say, 1823 e Sargus bipunctatus (Scopoli, 1763). ROZKOŠNÝ (1982) redescreveu as larvas e pupários de duas espécies ocorrentes nas regiões Paleártica e Neártica (S. bipunctatus e Sargus cuprarius Linnaeus, 1758) e a larva e o pupário de Sargus flavipes Meigen, 1822 e Sargus iridatus (Scopoli, 1763), ambas ocorrentes na região Paleártica. Não existem dados sobre a descrição de larvas de espécies deste gênero para a região Neotropical, o que alerta para a necessidade de estudos sobre a biologia e taxonomia das larvas deste grupo.

Neste trabalho é realizada a descrição do pupário de $S$. thoracicus.

\section{MATERIAL E MÉTODOS}

Uma larva de $S$. thoracicus foi coletada em compostagem no setor dos alojamentos estudantis do campus da Universidade Federal Rural do Rio de Janeiro (UFRRJ). A larva foi coletada com auxílio de uma pinça, acondicionada em pote plástico e levada para o laboratório onde foi acondicionada em placa de Petri até a emergência do adulto, seguindo a metodologia para criação de larvas de acordo com Pujol-Luz \& Xerez (1999) e Pujol-Luz \& Leite (2001). Após a eclosão do adulto, o pupário foi colocado numa solução de ácido hidroclorídrico a 10\% para retirada de detritos incorporados no tegumento, seguindo a técnica descrita em NOTMAN (1922). O pupário foi fixado em álcool a 70\% para posterior descrição.

Para a confecção dos desenhos foi utilizado um microscópio estereoscópico Wild M5 acoplado com câmara clara. Para os desenhos da cabeça e tórax (ventral e dorsal) foi utilizado aumento de 50X e do abdômen (ventral e dorsal) foi utilizado o aumento de $24 \mathrm{X}$.

A terminologia adotada na descrição do pupário seguiu ROZKOŠNÝ (1982) e PUJOL-LuZ \& LeITE (2001). O material utilizado no trabalho encontra-se depositado na Coleção Entomológica Costa Lima (CECL) do Instituto de Biologia da UFRRJ.

\section{RESULTADOS}

\section{Sargus thoracicus Macquart, 1834}

Figs 1-6

Distribuição. Neotropical: México, Guatemala, Honduras, Costa Rica, Panamá, Brasil (Rio de Janeiro).

Pupário. Comprimento: 7,9 mm. Cabeça projetada anteriormente, corpo alongado e cilíndrico, recoberto por placas cuticulares de formato hexagonal. Corpo com placas cuticulares mais esclerotizadas delimitando os segmentos e nas margens 
laterais do corpo. Coloração castanho claro, com três faixas longitudinais mais escuras partindo do primeiro segmento torácico e terminando no oitavo segmento abdominal (Fig. 1).

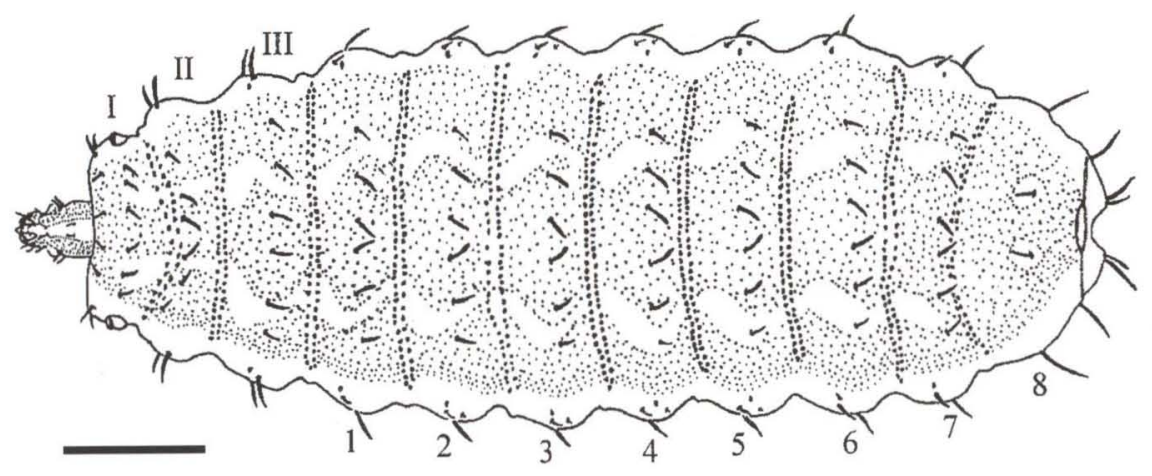

Fig.1. Habitus do pupário de $S$. thoracicus, vista dorsal. Barra de escala $=1 \mathrm{~mm}$.

Cabeça sub-retangular, projetada anteriormente, tendo uma região mais escura nas margens laterais e na região anterior. Olhos arredondados localizados na região mediana. Duas faixas transversais mais escuras, partindo do labro e terminando na parte posterior da cabeça. Antenas curtas, bissegmentadas, localizadas no terço apical. Quetotaxia: dois pares de cerdas labrais (Lb), dois pares de cerdas clípeo-frontais (cf), um par de cerdas dorsolaterais (Dl) localizados acima dos olhos e um par de cerdas laterais (L) localizadas abaixo dos olhos (Fig. 2); três pares de cerdas ventrolaterais (Vl) e dois pares de cerdas ventrais (V) (Fig. 3). Complexo mandíbulo-maxilar esclerotinizado e não funcional.

Tórax. Primeiro segmento mais curto do que os outros; espiráculos anteriores localizados na região mediana do segmento, de forma semi-circular com uma região mais escura no terço apical anterior; duas fileiras de cerdas na região dorsal e uma fileira na região ventral. Quetotaxia: dois pares de cerdas anterodorsais (Ad), um par de cerdas dorsolaterais (Dl) e três pares de cerdas dorsais (D) (Fig. 2); dois pares de cerdas ventrais (V), sendo o par mais externo bifurcado e um par de cerdas ventrolaterais (Vl) (Fig. 3). Segundo segmento mais curto do que o terceiro, sendo que ambos apresentam três pares de cerdas dorsais (D) e um par de cerdas laterais (L) (Fig. 1). Em vista ventral apresentam dois pares de cerdas ventrais (V) sendo o par mais externo bifurcado e um par de cerdas ventrolaterais (Vl).

Abdômen. Segmentos de 1-6 com a mesma forma, sétimo segmento mais curto do que os outros (Fig. 1). Cerdas dorsais externas de todos segmentos abdominais de menor tamanho. Quetotaxia: primeiro e sexto segmentos abdominais com três pares de cerdas dorsais (D), um par dorsolateral (Dl), um par lateral (L), três pares de cerdas ventrais (V) e um par de cerdas ventrolateais (Vl); segundo ao quinto segmento sem a cerda lateral e apresentando os espiráculos pupais; sétimo 

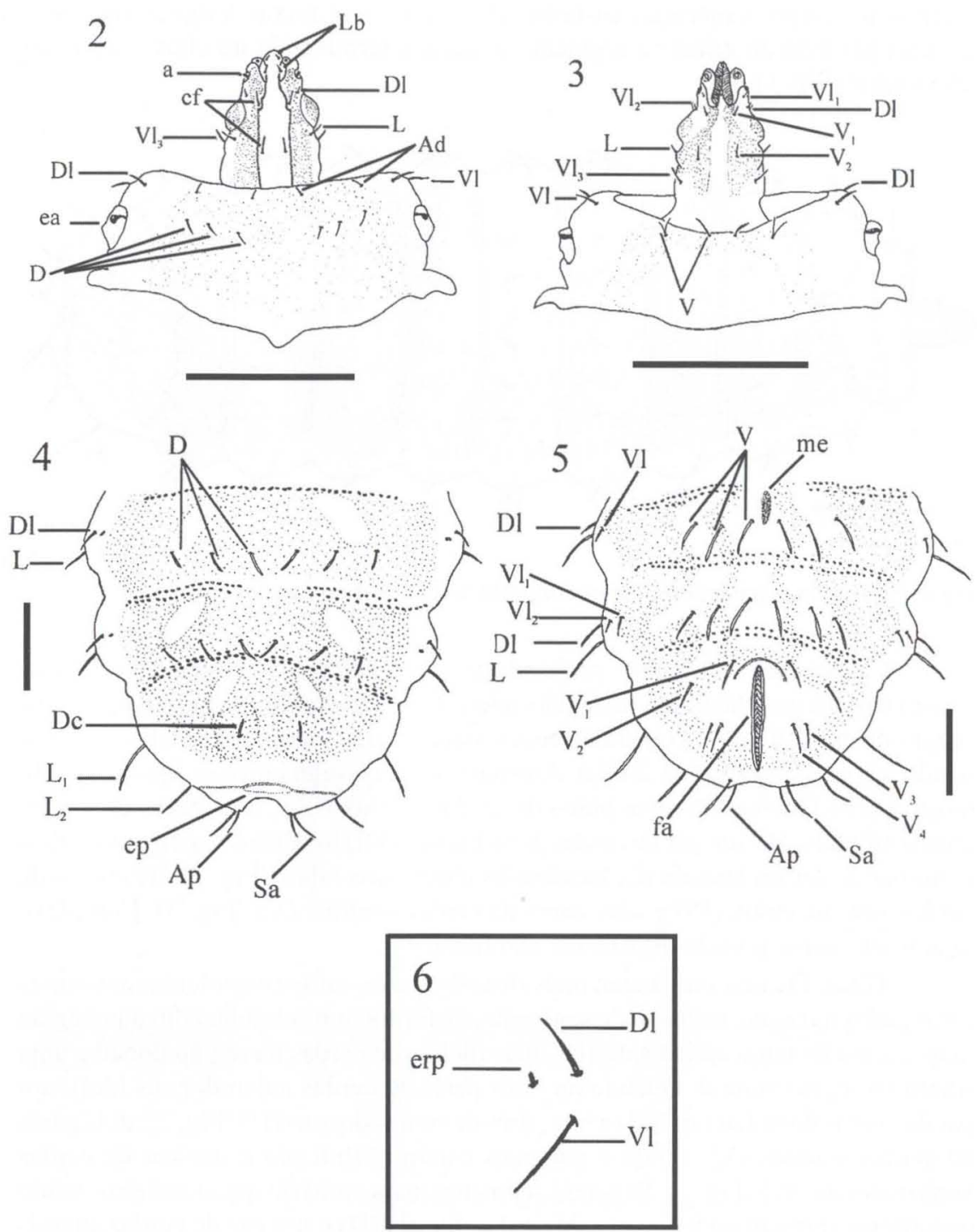

Figs 2-6. Sargus thoracicus: (2) cabeça e primeiro segmento torácico em vista dorsal; (3) cabeça e primeiro segmento torácico em vista ventral; (4) segmentos abdominais 6-8, vista dorsal; (5) segmentos abdominais 6-8, vista ventral; (6) esquema da região lateral do segmento abdominal 3. Barra de escala $=1 \mathrm{~mm}$. (a) Antena, (Ad) cerdas anterodorsais, (Ap) cerdas apicais, (cf) cerdas clipeofrontais, (D) cerdas dorsais, (Dc) cerdas dorsocentrais, (DI) cerdas dorsolaterais, (ea) espiráculo anterior, (ep) espiráculo posterior, (erp) espiráculo respiratório pupal, (fa) fenda anal, (Lb) cerdas labrais, (L) cerdas laterais, (me) mancha esternal, (As) cerdas subapicais, $(\mathrm{V})$ cerdas ventrais, (VI) cerdas ventrolaterais. 
segmento com dois pares de cerdas ventrolaterais (Vl); oitavo segmento com um par de cerdas dorsocentrais (Dc), dois pares de cerdas laterais, um par de cerdas apicais (Ap) e um par de cerdas subapicais (Sa) (Fig. 4). Sexto segmento com mancha esternal (me) de forma elíptica (Fig. 5). Oitavo segmento abdominal de forma semi-elíptica com uma constricção mediana e espiráculo posterior (ep) abrindo-se na região posterior do segmento (Fig. 4). Fenda anal (fa) na região ventral, rodeada por quatro pares de cerdas ventrais (V) (Fig. 5).

\section{DISCUSSÃO}

Na minicompostagem, além da larva de $S$. thoracicus, foram encontradas larvas de quatro espécies de Stratiomyidae: Hermetia illucens Linnaeus, 1758, Microchysa bicolor Wiedemann, 1830, Ptecticus testaceus (Fabricius), 1805 e Merosargus cingulatus Schiner, 1868. As larvas de H. illucens (Hemetiinae) ocorreram em maior quantidade; os adultos parecem ter preferência por este tipo de matéria orgânica (restos de frutas e verduras em decomposição) para a postura de ovos, podendo ainda fazer suas posturas em cadáveres de animais (MCFADDEN 1967). Larvas de P. testaceus e M. cingulatus (Sarginae) foram encontradas em menor quantidade na minicompostagem, sendo que as larvas destas duas espécies foram coletadas em abundância por PUJOL-LuZ \& LEITE (2001) no fruto em decomposição do abricó-de-macaco, Couroupita guianensis Aubl. 1775 (Lecythidaceae). Larvas de $M$. bicolor (Sarginae) também foram encontradas em grande quantidade na minicompostagem, mas precisam de maior atenção para serem coletadas devido ao seu pequeno tamanho $(0,55-065 \mathrm{~mm})$.

MCFADDEN (1967) forneceu dados sobre a biologia, descreveu e elaborou uma chave para identificação de quatro espécies de Sargus. Larvas de S. elegans foram coletadas em esterco de cavalo; $S$. decorus foram coletadas em folhas apodrecidas e em esterco de vaca e as larvas de $S$. bipunctatus e $S$. cuprarius foram coletadas em esterco bovino. As descrições fornecidas por MCFADDEN (1967) foram sucintas, baseando-se na coloração da larva e posição do espiráculo protorácico não se atendo a quetotaxia das larvas.

ROZKOŠNÝ (1982) redescreveu as larvas e pupários de três espécies de Sargus ocorrentes na Europa (S. bipunctatus, S. flavipes, S. cuprarius) e descreveu a larva e o pupário de $S$. iridatus, fornecendo uma chave para identificação destas espécies.

A seguir, compara-se as diferenças encontradas no pupário de $S$. thoracicus com as larvas e pupários das espécies estudadas por ROZKOŠNÝ (1982): S. thoracicus difere do pupário de $S$. cuprarius pela forma da cabeça, sub-retangular em $S$. thoracicus e cônica em $S$. cuprarius; $S$. thoracicus difere de $S$. bipunctatus por apresentar quatro bandas de cores amareladas no corpo, S. bipunctatus apresenta seis bandas de cores amareladas na região ventral e dorsal do corpo, as cerdas da cabeça de $S$. bipunctatus são mais longas do que as de $S$. thoracicus; $S$. thoracicus difere de $S$. flavipes por apresentar a margem ocular curta; $S$. thoracicus difere de S. iridatus pelo tamanho, S. thoracicus tem 7,9 mm e $S$. iridatus varia de $10-12,8$ $\mathrm{mm}$. Duas características relevantes do pupário de S. thoracicus são a perda de uma cerda ventral da região cefálica e a perda de um par de cerdas ventrolaterais. 
AGRADECIMENTOS. Aos meus pais, ao Dr. José Ramos Glória e ao biólogo Alexandre Ururahy Rodrigues pelo incentivo e apoio na realização da pesquisa (GGV). A Taila da Silva Guimarães, pelas sugestões e correções gramaticais (GGV). Ao Programa Institucional de Bolsas de Iniciação Científica da Universidade Federal Rural do Rio de Janeiro (GGV).

\section{REFERÊNCIAS BIBLIOGRÁFICAS}

James, M.T. 1981. Stratiomyidae, p. 497-511. In: J.F. McAlpine; B.V. Peterson; G.E. Shewell; H.J. TESkey; J.R. Vockeroth \& D.M. Wood (Eds). Manual of Neartic Diptera. Agriculture Canada, Ottawa, vol. 1, I-VI+674.

KovaC, D. \& R. RozkošNÝ. 1997. Contributions to the taxonomy and biology of Malasian soldier flies

(Diptera, Stratiomyidae). Folia Fac. Sci. Nat. Univ., Brno, 8: 89-92.

McFadden, M.W. 1967. Soldier fly larvae in America North of Mexico. Proceed. Unit. Stat. Nat. Mus. 121: $1-72$.

Notman, H. 1922. Stratiomiid larvae and puparia of the North Eastern states. Jour. N.Y. Entomol. Soc. 30: $141-153$.

Pujol-Luz, J.R. \& F.M. LeITE. 2001. Descrição do último ínstar larval e do pupário de Ptecticus testaceus

(Fabr.) (Diptera: Stratiomyidae). Neotrop. Entomol. 30 (4): 587-591.

Pujol-Luz, J.R. \& F.D. Vieira. 2000. A larva de Chiromyza vittata Widemann (Diptera: Straiomyidae).

An. Soc. Entomol. Brasil 29 (1): 49-55.

Pujol-LuZ, J.R. \& R. DE XEREZ. 1999. The larva of Chalcidomorphina aurata Enderlein 1914 (Diptera:

Stratiomyidae) from "Ilha de Marambaia" Rio de Janeiro, Brazil. Proc. Entomol. Soc. Wash. 101

(2): 295-299.

RozkošnÝ, R. 1982. A Biosystematic Study of the European Stratiomyidae (Diptera). Volume 1.

Introduction, Beredinae, Sarginae and Stratiomyinae. London, W. Junk, VIII+401p.

Woodley, N.E. 2001. A World Catalogue of the Stratiomyidae (Insecta: Diptera). Myia (11): 1-473.

Recebido em 28.VIII.2002; aceito em 08.XI.2002. 